\title{
Manejo orgánico de la milpa: rendimiento de maíz, frijol y calabaza en monocultivo y policultivo
}

\author{
Organic milpa: yields of maize, beans, and squash in mono-and polycropping systems
}

\section{Roland Ebel $^{1 *}$, José Gonzalo Pozas Cárdenas ${ }^{1}$, Florencio Soria Miranda ${ }^{1}$ y Jesús Cruz González ${ }^{1}$}

${ }^{1}$ Universidad Autónoma del Estado de México, Facultad de Ciencias Agrícolas. Instituto Literario 100, Col. Centro. 50000, Toluca, Estado de México, México.

*Autor responsable (roland.ebel@gmx.com)

\section{RESUMEN}

La milpa es un tradicional policultivo Mesoamericano que incluye maíz (Zea mays L.), calabaza (Cucurbita spp.) y frijol (Phaseolus vulgaris L.). Se caracteriza por una sinergia entre estos tres cultivos que favorece su rendimiento en conjunto y genera resiliencia ante perturbaciones externas. No obstante, retos actuales ambientales y socio-económicos disminuyen el atractivo de la milpa para los productores por lo que es necesario innovar este sistema de producción sin perder su principal fortaleza, la agrobiodiversidad. En un experimento implementado en 2015 en Piedras Blancas, Estado de México, se compararon todas las posibles combinaciones de policultivos de maíz, calabaza y frijol con los monocultivos de cada una de estas plantas. Todo el manejo fue orgánico y la nutrición se basaba en estiércol compostado de bovino. De todos los tratamientos investigados, la asociación de maíz y frijol demostró la mayor producción total, donde el maíz rindió $7.9 \mathrm{Mg} \mathrm{ha}^{-1} \mathrm{y}$ el frijol $1.9 \mathrm{Mg} \mathrm{ha}^{-1}$. En este policultivo, cada planta de maíz produjo $68.3 \mathrm{~g}, 1.2$ veces superior al rendimiento por planta en monocultivo; y cada planta de frijol $27.5 \mathrm{~g}$, lo que corresponde a su rendimiento en el monocultivo. Todas las demás combinaciones de cultivos (maíz-calabaza, frijolcalabaza y maíz-frijol-calabaza) superaron también los respectivos monocultivos en cuanto a su rendimiento total. Con respecto a la calabaza se destacó que las diversas asociaciones incrementaron su rendimiento por planta, pero disminuyeron la producción por planta de maíz y frijol asociados con la calabaza. Se confirmó la sobrecosecha de maíz y frijol asociados comparado con los respectivos monocultivos, lo que subraya

Cita recomendada:

Ebel, R., J. G. Pozas Cárdenas, F. Soria Miranda y J. Cruz González. 2017. Manejo orgánico de la milpa: rendimientos de maíz, frijol y calabaza en monocultivo y policultivo. Terra Latinoamericana 35: 149-160. el potencial productivo de los sistemas de producción diversificados.

Palabras clave: agrobiodiversidad, agricultura tradicional, Estado de México, sobrecosecha.

\section{SUMMARY}

The milpa is a traditional Mesoamerican production system based on polycropping corn (Zea mays L.), squash (Cucurbita spp.), and bean (Phaseolus vulgaris L.). Milpa is characterized by synergetic interactions between these plants that favor the total output of this arrangement and increase resilience to perturbations of the entire agroecosystem. Still, current environmental and socio-economic threats decrease the milpa's attractiveness for farmers. Therefore, innovations of milpa are necessary but should not affect its principal strength: its agrobiodiversity. In Piedras Blancas, central Mexico, in 2015, an experiment was carried out to compare all possible combinations of maize, squash, and bean polyculture. Crop management was based on the principles of organic farming and fertilization was provided using manure compost. The combination of maize and beans achieved higher total production, in which maize yielded $7.9 \mathrm{Mg} \mathrm{ha}^{-1}$ and beans $1.9 \mathrm{Mg} \mathrm{ha}^{-1}$. In this maize-bean polyculture, each maize plant produced $68.3 \mathrm{~g}, 1.2$ times higher than the yield per plant in monoculture and each bean plant yielded $27.5 \mathrm{~g}$, which was equal to its performance in monocropping. All other combinations of crops (maizesquash, bean-squash, and maize-bean-squash) also exceeded their respective monocultures in terms of total production. As regards squash, it was revealed that the diverse associations increased its yield per plant, but per

Recibido: agosto de 2016. Aceptado: enero de 2017. Publicado en Terra Latinoamericana 35: 149-160. 
plant production of maize and beans decreased when they were associated with squash. The overyielding of maize and beans in association, compared with their respective monocultures, highlights the potential of polycropping systems to provide satisfactory yields for all involved crops.

Index words: agrobiodiversity, traditional farming, State of Mexico, overyielding.

\section{INTRODUCCIÓN}

La milpa es un sistema tradicional de policultivo que tiene su origen en Mesoamérica (Hernández, 1995); consiste en la asociación simultanea de maíz (Zea mays L.), calabaza (Cucurbita argyrosperma Huber, C. ficifolia Bouché, C. moschata Duchesne ex Poir o $C$. pepo L.) y diversas leguminosas (Phaseolus vulgaris L., P. lunatus L., Vicia faba L. o Vigna unguiculata [L.] Walp.), cultivos que en esta parte del mundo se conocen como las tres hermanas (Aguilar et al., 2003; Lewandowski, 1987). Aunque existen regiones en México y Centroamérica donde la palabra milpa se refiere a cualquier terreno dedicado al cultivo del maíz (Real Academia Española, 2014), en el presente artículo, milpa se refiere únicamente al mencionado policultivo.

En el caso de la milpa se trata de un policultivo mixto donde varios cultivos crecen simultáneamente sin un arreglo por surcos (Gliessman, 1985). La milpa es además un policultivo suplementario, donde se reduce la densidad de siembra del cultivo principal (en el caso de la milpa, el maíz) para dejar espacio para uno o varios cultivos asociados. Se distingue de policultivos aditivos, donde la densidad de siembra del cultivo principal se mantiene constante (Iverson et al., 2014).

Según el clima, el suelo, la vegetación, las costumbres alimenticias de la región y los intereses y destrezas del productor, la milpa puede incluir un sinfín de plantas (anuales y perennes) adicionales. Pueden llegar a encontrarse hasta 50 especies diferentes ya sea cultivadas, auspiciadas o toleradas (Aguilar et al., 2003; Molina et al., 2016). Frecuentemente, un maíz nativo con un ciclo de producción corto es intercalado con otro de un ciclo mayor. Los agricultores, muchos de ellos en subsistencia, mantienen esta diversidad inter e intraespecífica como estrategia para enfrentar futuros cambios ambientales y necesidades económicas actuales (Altieri, 2009a). La milpa se desarrolla tradicionalmente en temporal $\mathrm{y}$ sin mayor involucramiento de agromaquinaria. La agrobiodiversidad generada es la principal estrategia para enfrentar plagas y enfermedades. En muchas partes de México, el manejo de barbecho en la milpa es relacionado con la práctica de roza, tumba y quema que también aporta nutrientes a estos policultivos (Mariaca, 2011). En el centro de México, comúnmente se presenta la milpa con insumos de sistemas de producción convencionales (p. ej.: nutrición vegetal con fertilizantes sintéticos o uso de plaguicidas).

La interacción de maíz, leguminosas y calabaza genera un aumento de la producción por planta de maíz en comparación con su monocultivo, la sobrecosecha (Altieri et al., 2011). El rendimiento de un cultivo es altamente determinado por su competencia intraespecífica; en un policultivo suplementario, una sustitución parcial del cultivo principal por otro incrementa su nivel de producción por una reducción de la competencia intraespecífica (Iverson et al., 2014). Gliessman (1985) demostró que 1.73 ha de maíz en monocultivo producen tanto alimento como 1 ha de milpa; generando una cantidad adicional de 2 $\mathrm{Mg} \mathrm{ha}^{-1}$ de residuos de cultivo (para ser incorporados al suelo) en comparación con un monocultivo. Según Zhang et al. (2014), el policultivo de maíz y frijol (sin calabaza) genera una sobrecosecha parecida.

Sobrecosecha en un policultivo como la milpa se debe a (1) efectos de selección donde prospera el cultivo dominante gracias a aportaciones de los demás o a (2) complementariedad de nicho donde las diversas plantas aprovechan nichos diferentes. En la milpa se detectan ambos fenómenos (Zhang et al., 2014) pero predomina la complementariedad de nicho causada por diferencias arquitectónicas de las raíces entre maíz, frijol y calabaza que resulta en una eficiente absorción de nutrientes (Postma y Lynch, 2012). Aparte, el frijol es una planta fijadora de nitrógeno atmosférico que aporta este nutriente a los demás cultivos. En cambio, la caña de maíz proporciona sostén al frijol. La calabaza sembrada entre el maíz y el frijol cubre ampliamente el suelo y consecuentemente limita el desarrollo de arvenses y ayuda a mantener la humedad del suelo (Aguilar et al., 2003; Gliessman, 1990; Wang et al., 2010).

La milpa se considera un sistema de producción tradicional, donde los rendimientos de los cultivos dependen de los recursos internos, el reciclaje de 
materia orgánica, los mecanismos de control biológico y el patrón de lluvia (Altieri, 2009a). Sin embargo, desde la segunda parte del siglo XX, en México, las políticas gubernamentales promovieron el monocultivo y la utilización de estrategias como la mecanización, el mejoramiento de variedades y el desarrollo de agroquímicos para la fertilización y el control de plagas y arvenses. Desde entonces, la agricultura quedó al servicio de la industrialización (Aguilar et al., 2003). Como consecuencia, los ciclos de nutrientes, energía y agua en los monocultivos se han vuelto más abiertos que en los ecosistemas tradicionales y la eficiencia del uso de insumos ha disminuido; hoy en día, los rendimientos en la mayoría de los cultivos importantes se están estancando (Altieri, 2009a) y el tratamiento de pérdida de fertilidad, retención de agua, plagas y enfermedades requiere un incremento de los costos externos (Gliessman, 1977).

No sorprende, pues, que en México todavía persiste la milpa. Aun así, este sistema de producción presenta también desventajas por las cuales cada vez menos jóvenes optan por dedicarse a la milpa: una alta demanda de mano de obra, poco potencial de mecanización y un menor rendimiento por área que monocultivos de maíz basados en altos insumos (Ebel y Castillo, 2012; Molina et al., 2016). Adicionalmente, las presiones socioeconómicas y ambientales que enfrentan muchos milperos han ocasionado la reducción del período de barbecho, haciendo menos productivo y sustentable este sistema (Rosales et al., 2004 ${ }^{1}$ ).

Otra amenaza para la milpa es el cambio climático. Para Mesoamérica, se espera para la mitad del siglo XXI que la producción de maíz disminuirá al menos en un 10\% (Jones y Thornton, 2003). En México, el cambio climático afecta particularmente a los agricultores en subsistencia cuya seguridad alimentaria depende del cultivo de maíz en temporal (Altieri, 2009b). Se pronostican múltiples consecuencias directas del cambio climático al desarrollo, la productividad y la fenología de los cultivos: en primer lugar un daño físico de los agroecosistemas causado por un incremento en desastres naturales (Morton, 2007); adicionalmente, un aumento de la actividad fotosintética de las plantas por las esperadas temperaturas y niveles de $\mathrm{CO}_{2}$ elevados, fomentando la biomasa de las arvenses (Führer, 2003; Patterson, 1995); una más intensa mineralización causada por una reproducción acelerada de la microflora del suelo (Pimentel, 1993; Swaminathan y Kesavan, 2012). También se pronostica una alteración de las poblaciones de hongos y bacterias patógenos (Biggs y Webb 1986; Dwivedi et al., 2013), menor actividad de micorrizas (Augé, 2001) y un incremento de ciclos reproductivos de insectos plaga (Altieri y Koohafkan, 2008).

En cuanto a los cultivos que componen la milpa, Phaseolus spp. es el más afectado, especialmente por períodos de sequía cuando tiene que invertir más energía en su crecimiento vegetativo para poder desarrollar un sistema radicular suficientemente profundo para extraer humedad del suelo (Dwivedi et al., 2013). Con respecto al maíz, su rendimiento puede disminuir hasta un $20 \%$ si los productores latinoamericanos siguen enfrentando una disminución de las precipitaciones (Dempewolf et al., 2014). La sequía afectará también su calidad; se espera una reducción de proteínas y un aumento de fibra, almidón y azúcar (Ali y Ashraf, 2011). Para Brassicaceas, una sequía prolongada resulta en una floración precoz, así como en descendientes con tallos más delgados (Dwivedi et al., 2013). Cabe mencionar que la mayoría de los estudios relacionados con las consecuencias del cambio climático se refiere al manejo de monocultivos.

Por el contrario, los agroecosistemas tradicionales se caracterizan por una biodiversidad funcional que genera sinergismos que garantizan una resiliencia ante perturbaciones externas y un alto potencial de adaptación a situaciones cambiantes (Altieri, 2004). Cuando más complejo y diverso sea un agroecosistema, menos es la fluctuación en términos de diversidad de especies lo que fomenta la estabilidad del sistema (Sullivan, 1998). Esta virtud de los policultivos depende del nivel de interacción entre los componentes bióticos, domesticados y salvajes, y abióticos de un agroecosistema (Altieri, 2009a) y del manejo de los productores (Altieri, 2009b). Consecuentemente, el policultivo, la agrobiodiversidad implementada, destaca por ser una estrategia eficiente para enfrentar los retos del cambio climático.

Aparte de su alta resistencia a perturbaciones, otras ventajas de los policultivos incluyen: bajo riesgo de una pérdida completa de la cosecha en caso de una perturbación (Ebel et al., 2013); la utilización adecuada del tiempo por el productor, ya que no todos los cultivos se desarrollan simultáneamente (Aguilar et al., 2003); correspondientemente, un periodo más extenso de cosecha; una variedad de alimentos producidos para productores en subsistencia; un aprovechamiento eficiente del área disponible

\footnotetext{
${ }^{1}$ Rosales G., M., I. Solís F. y A. Ayala S. 2004. Problemática campesina, retos y perspectivas de la investigación y el servicio para el mejoramiento de la milpa en Yucatán. De los maiceros, necesidades y expectativas. Memoria de Trabajos y Resultados del Foro Taller. INIFAP, INAH. Mérida, México.
} 
(Gliessman, 1985); un uso eficiente de luz, agua y nutrientes disponibles siempre que se asocien cultivos con diferentes fisionomías (Liebman, 1997; Bavec et al., 2005; Wang et al., 2010); alta disponibilidad de nitrógeno en el suelo cuando se intercalan leguminosas (Agboola y Fayemi, 1971); mayor captura de luz por tener más superficie foliar que en monocultivos; como consecuencia, mayor producción de biomasa (Zhang et al., 2014); la supresión biológica de enfermedades (Root, 1973; Altieri, 1994; Guzman y Alonso, 2000; Khan et al., 2008); una mayor actividad biológica de los suelos comparado con monocultivos (Gliessman, 1985); y asiduamente una amplia cobertura del suelo, reduciendo la erosión, el crecimiento de arvenses y la evaporación de agua (Ebel et al., 2013).

Las desventajas de los policultivos son su baja mecanización (Molina et al., 2016) e investigación al respecto; consecuentemente, una alta demanda de mano de obra (Frison et al., 2011); una falta de expertos en el área; una contracorriente social, política y económica; una difícil aplicación de métodos estadísticos convencionales para evaluar experimentos biodiversos; competencia entre los cultivos por nutrientes, luz y agua si los policultivos son mal diseñados; posibles interacciones alelopáticas entre las plantas; y una alta humedad relativa que puede fomentar mayor presencia de hongos patógenos (Gliessman, 1985).

A pesar de las múltiples fortalezas de los policultivos, la magnitud del cambio climático reta a los productores tradicionales de una manera que reaccionan con medidas como la disminución de la agrobiodiversidad que ponen en peligro su capacidad de resiliencia y adaptación. Es probable que la milpa tradicional no es capaz de enfrentar este reto, sino que requiere de una adaptación a los retos del siglo XXI sin perder sus características: su agrobiodiversidad y baja dependencia de insumos externos. Proponemos, pues, desarrollar agroecosistemas basados en la agricultura tradicional, adaptados a las condiciones climáticas y edáficas de un determinado lugar, pero enriquecidos por técnicas contemporáneas del manejo sostenible. Consecuentemente, la presente investigación busca aportar insumos para el desarrollo de un sistema de producción milpero adaptado a las condiciones ambientales del Estado de México, combinando el manejo tradicional con estrategias de la agricultura orgánica. Su objetivo fue encontrar el policultivo más productivo. Por lo tanto, se compararon todas las posibles combinaciones de policultivos de las plantas que componen la milpa (maíz, frijol, calabaza) con los monocultivos de cada una de estas especies, evaluando su rendimiento.

\section{MATERIALES Y MÉTODOS}

El experimento se realizó en el Campus El Cerrillo Piedras Blancas de la Universidad Autónoma del Estado de México, a $18 \mathrm{~km}$ al norte de la ciudad de Toluca, y a $55 \mathrm{~km}$ del sur de la ciudad de Atlacomulco. Se ubica a $19^{\circ} 14^{\prime} \mathrm{N}$ y $92^{\circ} 42^{\prime} \mathrm{O}$, a una altitud de $2611 \mathrm{~m}$. El clima prevaleciente es C $\left(\mathrm{w}_{2}\right)$ (García y Falcón, 1980). En la ciudad de Almoloya de Juárez, ubicada a $9.5 \mathrm{~km}$ del lugar del experimento, la temperatura media es de $13.3^{\circ} \mathrm{C}$, la precipitación anual de $744 \mathrm{~mm}$ (CONAGUA, 2016). Se trata de una zona templada subhúmeda donde las heladas anuales representan una limitación ambiental para la agricultura. En esta zona, la mayor parte de la producción de maíz es de temporal; el riego es generalmente de auxilio. Tradicionalmente, se siembran variedades nativas de maíz blanco, negro, amarillo y rosado, destacando 'Cacahuacintle' y 'Palomero Toluqueño' (Aguilar et al., 2003).

El experimento se implementó en un área de una extensión total de $201.6 \mathrm{~m}^{2}$. El diseño experimental fue de bloques completos al azar con siete tratamientos (de $7.2 \mathrm{~m}^{2}$ cada uno) y cuatro repeticiones de cada uno, resultando 28 unidades experimentales donde los tres cultivos experimentados se sembraron en diferentes densidades de siembra. Según recomendaciones de productores de la región, se sembraron 8 plantas de maíz $\mathrm{m}^{-2}$. La composición de los policultivos se calculó de la siguiente manera: la siembra de una planta de maíz equivale a 0.75 plantas de frijol y a 0.25 plantas de calabaza.

Las semillas de maíz, frijol y calabaza utilizadas en este experimento procedieron de la comunidad de El Rincón de la Candelaria (altitud de $2570 \mathrm{~m}$ ), municipio de Atlacomulco, México. Estos materiales fueron proporcionados por productores cooperantes que han sembrado dichos cultivos por muchos años obteniendo buenos resultados. La siembra del maíz se llevó a cabo el 25 de abril de 2015, utilizándose semilla de maíz nativo localmente conocido como 'Criollo blanco'. Esta variedad es localmente apreciada por una amplia resistencia al volcamiento, satisfactorias características de nixtamalización y buen sabor de su tortilla. Tres semanas después, se sembró frijol criollo 'Rosita' de mata y calabaza criolla de guía. 
El manejo del riego, de la nutrición y del control de plagas, enfermedades y arvenses fue idéntico para todos los tratamientos. El experimento se implementó principalmente en temporal. Únicamente en tres ocasiones se realizó riego por aspersores circulares: en virtud de bajas precipitaciones en el mes de abril, con la siembra de maíz se aplicó una cantidad de $500 \mathrm{~m}^{3} \mathrm{ha}^{-1}$; con la siembra de los otros cultivos el 16 de mayo de 2015 se aplicó la misma cantidad; un último riego de $350 \mathrm{~m}^{3}$ ha $^{-1}$ se realizó el 27 de mayo de 2015.

En cuanto a la nutrición vegetal, se aplicaron $10 \mathrm{Mg} \mathrm{ha}^{-1}$ estiércol de bovino con la siembra. Durante el desarrollo vegetativo de los diversos cultivos se emplearon $0.5 \mathrm{Mg} \mathrm{ha}^{-1}$ del fertilizante comercial orgánico "Naturabono". Se trata de un fertilizante producido a través del compostaje de residuos orgánicos urbanos y enriquecido con bacterias entomopatógenas, con un contenido de 1.1 (Vol.) $\% \mathrm{~N}, 0.7 \% \mathrm{~K}, 0.8 \% \mathrm{P}, 0.3 \% \mathrm{Mg}$, $3.1 \%$ Ca y $0.2 \% \mathrm{~S}$, aparte de trazas de Fe, Mn, Zn y B. Este producto se aplicó directamente al suelo donde se incorporó superficialmente. Adicionalmente, se aplicó quincenalmente un lixiviado de lombricomposta en dosis al equivalente de $7 \mathrm{~L} \mathrm{ha}^{-1}$ diluidos 1:4 en agua como fertilizante foliar (Cuadro 1). La aportación total de estas dos medidas corresponde a $165 \mathrm{~kg} \mathrm{ha}^{-1} \mathrm{~N}$, $95 \mathrm{~kg} \mathrm{ha}^{-1} \mathrm{P}$ y $245 \mathrm{~kg} \mathrm{ha}^{-1} \mathrm{~K}$ durante el ciclo del cultivo.

Las plagas que se presentaron a lo largo del ciclo vegetativo de los cultivos fueron Diabrotica undecimpunctata Mannerheim, D. longicornis Say, Macrodactylus sp., Phyllophaga sp., Spodoptera frugiperda Walker, Rhopalosiphum maidis Fitch, Chaetocnema pulicaria F. E. Melsheimer, Dalbulus maidis De Long, Helix aspersa Müller, Helicoverpa zea Boddie y Sphenophorus sp.; todas de baja incidencia. Preventivamente, se aplicó cal en los contornos del área experimental (en una ocasión), se fumigó un té de chile, ajo y cebolla (semanalmente con una dosis de $1.5 \mathrm{~L} \mathrm{ha}^{-1}$ ) y se instalaron trampas de cerveza. Aparte de insectos, se presentaron ocasionalmente roedores de las especies Rattus norvegicus Berkenhout y Rattus rattus L.

Las enfermedades que se observaron en baja incidencia fueron Puccinia sorghi en maíz, Fusarium sp. en frijol y Sphaerotheca sp. en la calabaza. No fue necesaria la aplicación de fungicidas orgánicos.

El manejo de arvenses fue manual a través de un deshierbe selectivo semanal. Las especies con mayor presencia fueron Medicago polymorpha L. (tolerada), Brassica napa L., Rumex crispus L., Lopezia racemosa Cav. y Commelina erecta L. (no toleradas). Su distribución fue pareja sobre todos los tratamientos. Lo mismo aplica para otras arvenses que se presentaron con menor frecuencia, tales como Calandrinia micrantha Schltdl., Bidens odorata Cav., Chenopodium album L. y Avena fatua $\mathrm{L}$.

El desarrollo vegetativo de las plantas fue monitoreado semanalmente. La cosecha de maíz se realizó en los meses de septiembre y octubre considerando la madurez fisiológica del cultivo. Para la medición del rendimiento, se seleccionaron al azar tres plantas del surco central de cada tratamiento que se secaron hasta alcanzar un promedio de 14\% de humedad, para posteriormente pesarlas y conocer su rendimiento. El frijol se cosechó en el mes de septiembre en cuanto alcanzó la madurez fisiológica y se dejó secar bajo sombra al aire libre hasta también alcanzar un $14 \%$ de humedad para pesarse posteriormente. La calabaza fue recolectada entre los meses de julio y agosto. Este cultivo se cosechó en forma de flor y fruto verde dos veces por semana hasta la senescencia de la planta; en cada corte, se pesó el fruto y se contó el número de flores.

Cuadro 1. Calendario de las principales actividades de nutrición y de control de plagas, implementadas el año 2015 en el campus El Cerrillo, Piedras Blancas de la Universidad Autónoma del Estado de México.

\begin{tabular}{|c|c|c|c|c|}
\hline Mes & Estiércol de bovino & Fertilizante comercial orgánico & Lixiviado de lombricomposta & Té de chile, ajo y cebolla \\
\hline Abril & $\bar{X}$ & & & \\
\hline Mayo & & & & $\mathrm{X}$ \\
\hline Junio & & $\mathrm{X}$ & $\mathrm{X}$ & $\mathrm{X}$ \\
\hline Julio & & & $\mathrm{X}$ & $\mathrm{X}$ \\
\hline Agosto & & & $\mathrm{X}$ & $\mathrm{X}$ \\
\hline Septiembre & & & & $\mathrm{X}$ \\
\hline
\end{tabular}


Se evaluó la producción por área y por planta. También se comparó la producción de maíz, frijol y calabaza en los diversos arreglos con sus rendimientos usuales en la región; la producción regional de un cultivo equivalió a un $100 \%$; y su rendimiento en los siete tratamientos experimentados se calculó en forma de puntos porcentuales basados en este valor (producción referenciada).

Adicionalmente, se calculó la razón equivalente de la tierra (RET). Corresponde al área en monocultivo que se requiere para generar la misma cosecha que en 1 ha del policultivo analizado (Altieri et al., 1983; Gliessman, 1985). Genera una base estandarizada para comparar diferentes arreglos en diferentes situaciones (Francis y Decoteau, 1993). Para obtener la RET, primero se calcula la relación entre el rendimiento de cada cultivo que compone un policultivo y su respectivo rendimiento en monocultivo. Después se suman las áreas equivalentes para cada cultivo que compone un policultivo como desglosado en la Ecuación 1:

$$
R E T=\sum_{i=1}^{n} \frac{C P_{i}}{C M_{i}}
$$

Donde $\mathrm{n}$ corresponde al número de cultivos asociados, $\mathrm{CP}$ al rendimiento por hectárea de un cultivo en policultivo y $\mathrm{CM}$ a la cosecha del mismo cultivo en monocultivo en la misma superficie y con un manejo idéntico. Una RET $>1$ expresa una ventaja productiva de un policultivo (Bavec et al., 2005).

La distribución normal de todos los datos fue comprobada con la prueba Shapiro-Wilk. La homogeneidad de las varianzas se evaluó con la prueba de Levene. Las significancias se detectaron con un análisis de varianza y una prueba post-hoc de Tukey $(P \leq 0.05)$ para cada parámetro analizado, los cuales fueron: producción total por área $\left(\mathrm{Mg} \mathrm{ha}^{-1}\right)$, producción por planta (g) y número de flores (en el caso de la calabaza).

\section{RESULTADOS Y DISCUSIÓN}

En cuanto al rendimiento por superficie, el maíz mostró mayor producción en monocultivo, donde también fue sembrado con mayor densidad (Cuadro 2). El frijol rindió más por superficie en monocultivo y asociado con el maíz (Cuadro 3). La producción de la calabaza por superficie fue mayor en asociación con
Cuadro 2. Rendimiento por superficie $\left(\mathrm{Mg} \mathrm{ha}^{-1}\right)$ y referenciado de maíz nativo en monocultivo, asociado con frijol, con calabaza y con frijol más calabaza.

\begin{tabular}{lcc}
\hline & Rendimiento & $\begin{array}{c}\text { Rendimiento } \\
\text { referenciado }\end{array}$ \\
\hline Maíz & $\mathrm{Mg} \mathrm{ha}^{-1}$ & $\%$ \\
Maíz-Frijol & $9.3 \mathrm{a}^{\ddagger}(0.2)^{\S}$ & 133 \\
Maíz-Calabaza & $7.9 \mathrm{~b}(0.3)$ & 113 \\
Maíz-Frijol-Calabaza & $7.9 \mathrm{~b}(0.9)$ & 113 \\
& $4.5 \mathrm{c}(0.7)$ & 65
\end{tabular}

Tomando como referencia un rendimiento de maíz de $7 \mathrm{Mg} \mathrm{ha}^{-1}(100 \%)$ como esperable en la región Atlacomulco, Estado de México. ${ }^{\star}$ Letras distintas en la misma columna indican diferencias significativas, según la prueba de Tukey $(P \leq 0.05) .{ }^{\S}$ Desviación estándar.

el frijol, evaluando tanto la producción de flores como de frutos (Cuadro 4). En cambio, maíz, frijol y calabaza mostraron su menor rendimiento por superficie en el policultivo de las tres hermanas.

Para generar alta RET, el reto es encontrar la combinación de densidades de siembra más productiva (Sullivan, 1998). Siguiendo el manejo tradicional de la milpa, los policultivos en la presente investigación mostraron un arreglo suplementario donde las densidades de siembra de cada cultivo fue menor que en sus respectivos monocultivos. Productores del norte del Estado de México recomiendan una relación de 0.75 plantas de frijol y de 0.25 plantas de calabaza por cada maíz sembrado, tal como lo implementado en esta investigación. Gliessman (1985) reporta en un experimento con 0.8 plantas de frijol y 0.07 plantas de calabaza sembradas por cada planta de maíz (o sea, ligeramente mayor densidad de frijol y drásticamente

Cuadro 3. Rendimiento absoluto $\left(\mathrm{Mg} \mathrm{ha}^{-1}\right)$ y referenciado de frijol en monocultivo, asociado con maíz nativo, con calabaza y con maíz nativo más calabaza.

\begin{tabular}{lcc}
\hline & Rendimiento & $\begin{array}{c}\text { Rendimiento } \\
\text { referenciado }\end{array}$ \\
\hline Frijol & $\mathrm{Mg} \mathrm{ha}^{\dagger}$ & $\%$ \\
Maíz-Frijol & $1.9 \mathrm{a}^{\ddagger}(0.4)^{\S}$ & 124 \\
Frijol-Calabaza & $1.9 \mathrm{a}(0.5)$ & 128 \\
Maíz-Frijol-Calabaza & $0.6 \mathrm{~b}(0.2)$ & 40 \\
\hline
\end{tabular}

Tomando como referencia un rendimiento de frijol de $1.5 \mathrm{Mg} \mathrm{ha}^{-1}(100 \%)$ como esperable en la región Atlacomulco, Estado de México. " Letras distintas en la misma columna indican diferencias significativas, según la prueba de Tukey $(P \leq 0.05)$. ${ }^{\S}$ Desviación estándar. 
Cuadro 4. Rendimiento absoluto $\left(\mathrm{Mg} \mathrm{ha}^{-1}\right)$ y referenciado de calabaza (frutos) y número de flores de calabaza por ha en monocultivo, asociado con maíz nativo, con frijol y con maíz nativo más frijol.

\begin{tabular}{lccc}
\hline & Rendimiento (frutos) & Rendimiento (frutos) referenciado $^{\dagger}$ & Rendimiento (flores) \\
\hline & Mg ha $^{-1}$ & $\%$ & Flores ha $^{-1}$ \\
Calabaza & $10.3 \mathrm{~b}^{\ddagger}(2.6)^{\S}$ & 86 & $1268 \mathrm{~b}(124)$ \\
Maíz-Calabaza & $7.3 \mathrm{c}(0.7)$ & 61 & $788 \mathrm{c}(76)$ \\
Frijol-Calabaza & $14.0 \mathrm{a} \mathrm{(1.0)}$ & 116 & $1531 \mathrm{a}(109)$ \\
Maíz-Frijol-Calabaza & $6.2 \mathrm{c}(1.9)$ & 62 & $1120 \mathrm{~b}(91)$ \\
\hline
\end{tabular}

† Tomando como referencia un rendimiento de calabaza de $12 \mathrm{Mg} \mathrm{ha}^{-1}(100 \%)$ como esperable en la región Atlacomulco, Estado de México. ${ }^{\ddagger}$ Letras distintas en la misma columna indican diferencias significativas, según la prueba de Tukey $(P \leq 0.05){ }^{\S}$ Desviación estándar.

menor densidad de calabaza que en el presente experimento) que generó una RET excepcional de 1.73. Este resultado se debe a un mayor rendimiento por planta de maíz en policultivo que en monocultivo (sobrecosecha), independiente de diferentes densidades de maíz en monocultivo comparadas.

En el presente experimento, la producción por superficie de maíz en el policultivo de las tres hermanas también generó sobrecosecha. Corresponde a un $48 \%$ de su producción en monocultivo (cuando la densidad de siembra del maíz era tan solo un 33\% del monocultivo). Correspondiente al frijol, la producción por superficie en el policultivo con maíz y calabaza correspondió a un $47 \%$ del rendimiento de frijol en monocultivo (con un tercio de densidad del monocultivo). Este resultado sugiere un potencial de incremento de la producción de maíz a expensas del frijol si el maíz se sembrara con mayor densidad: incrementando la densidad de maíz, la productividad por área del mismo aumenta mientras la de una leguminosa asociada disminuye (Francis y Decoteau, 1993).

Finalmente, la calabaza produjo un $60 \%$ de su rendimiento por superficie en este policultivo cuando su densidad de siembra era de un 33\% del monocultivo. Usualmente, la producción de calabaza en la milpa es menor que en monocultivo (Gliessman, 1985). Sin embargo, se encuentra cuatro especies diferentes de calabaza (cada una con múltiples variedades) en las milpas de México (Aguilar et al., 2003) que se distinguen por considerables diferencias morfológicas y productivas (Mera et al., 2011). Por lo tanto, la calabaza es probablemente el componente de la milpa donde la comparación con otros experimentos tenga el menor valor informativo.

Para poder equiparar los rendimientos de las tres hermanas, se evaluó el rendimiento por planta: el maíz mostró su mayor rendimiento por planta en asociación con el frijol (Figura 1); el frijol rindió más asociado con maíz y en monocultivo (Figura 2); y la calabaza tuvo su mayor rendimiento asociada con maíz y frijol a la vez (Figura 3).

Arreglos suplementarios tienen un efecto positivo al rendimiento por planta del cultivo principal en comparación con su monocultivo la que usualmente no es compensada por la competencia interespecífica entre los cultivos asociados. Para el maíz, esta sobrecosecha fue comprobada para climas tropicales y templados (Iverson et al., 2014). Esto explica por qué el rendimiento por planta del maíz fue superior al monocultivo en su asociación con el frijol y con la calabaza (Figura 1).

Destaca sobre todo una interacción mutuamente positiva entre maíz y frijol. Es congruente con la observación de Zhang et al. (2014) según la cual policultivos de maíz y frijol generan sobrecosecha gracias a una mayor producción de biomasa de ambos que en un monocultivo. Sin embargo, sobre todo en el caso del frijol, esta sobrecosecha depende altamente de la variedad sembrada (Santalla et al., 2001). En cambio Albino et al. (2015) observaron solo un efecto positivo al rendimiento por planta de maíz. Opuesto a la costumbre en México existe también el enfoque de ver el frijol como cultivo principal de esta asociación. Tanto Tembakazi y Lucas (2002) como Sullivan (1998) reportan de experimentos que generaron alta RET y donde la densidad de siembra del frijol superó la del maíz.

Un resultado inusual obtenido en esta investigación fue que se obtuvo el rendimiento más alto por planta de calabaza en el policultivo de las tres hermanas. Usualmente, la calabaza rinde menos en policultivos con maíz que en monocultivo. Una explicación es que, 


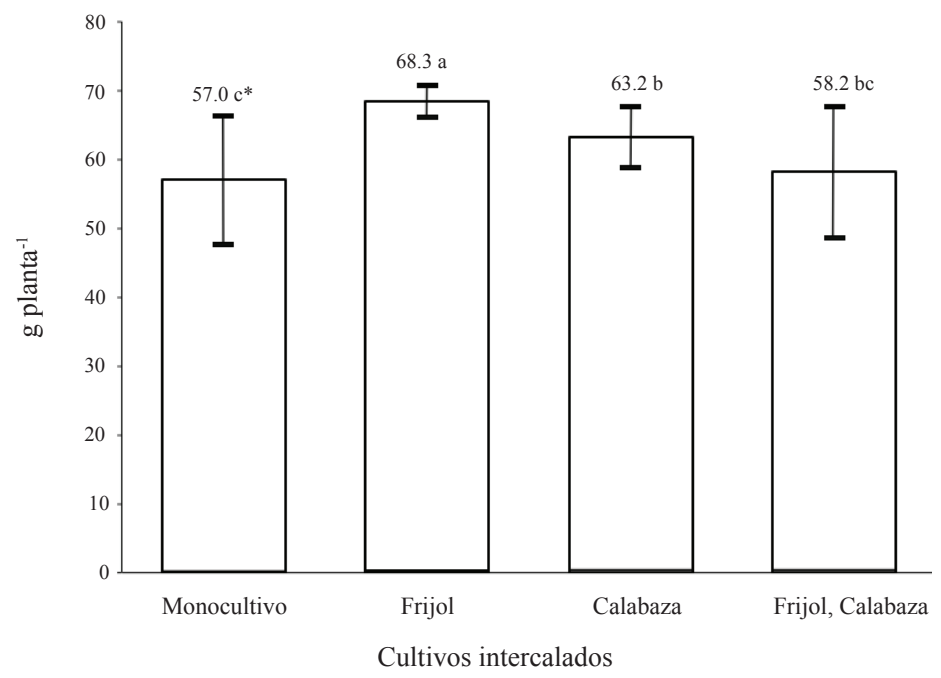

Figura 1. Rendimiento por planta de maíz en monocultivo, asociado con frijol, con calabaza y con ambos cultivos. * Letras distintas indican diferencias significativas, según la prueba de Tukey $(P \leq 0.05)$.

de las tres hermanas el rendimiento de la calabaza es el más dependiente de las condiciones atmosféricas (Risch y Hansen, 1982); y precisamente durante el ciclo productivo de la calabaza, se observó más altas precipitaciones de lo usual en el área del experimento (INIFAP, 2016) lo que fomenta la productividad de la calabaza criolla en este arreglo (Molina et al., 2016).

Según la evaluación de la RET, la combinación maíz-frijol resultó ser la más productiva. 1 ha de maíz y frijol mostró el mismo rendimiento como lo tuvieran 1.9 ha de estas plantas en monocultivo. A esta asociación, le siguen los intercalados maíz-frijolcalabaza y maíz-calabaza. El monocultivo de calabaza fue el tratamiento menos productivo. Destaca que todos los policultivos generaron una RET mayor a 1 (Cuadro 5).

Es comprobado que el policultivo maíz-frijolcalabaza con manejo adecuado genera sobrecosecha (Zhang et al., 2014). Una explicación son sinergias altamente funcionales entre maíz, frijol y calabaza

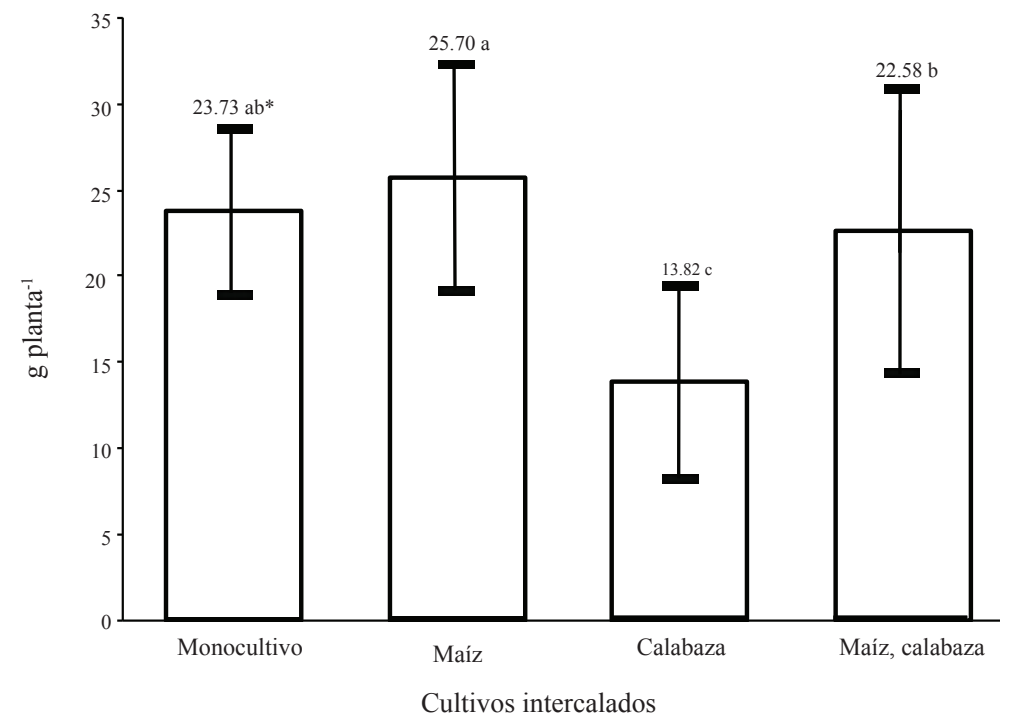

Figura 2. Rendimiento por planta de frijol en monocultivo, asociado con maíz, con calabaza y con ambos cultivos. * Letras distintas indican diferencias significativas, según la prueba de Tukey $(P \leq 0.05)$. 


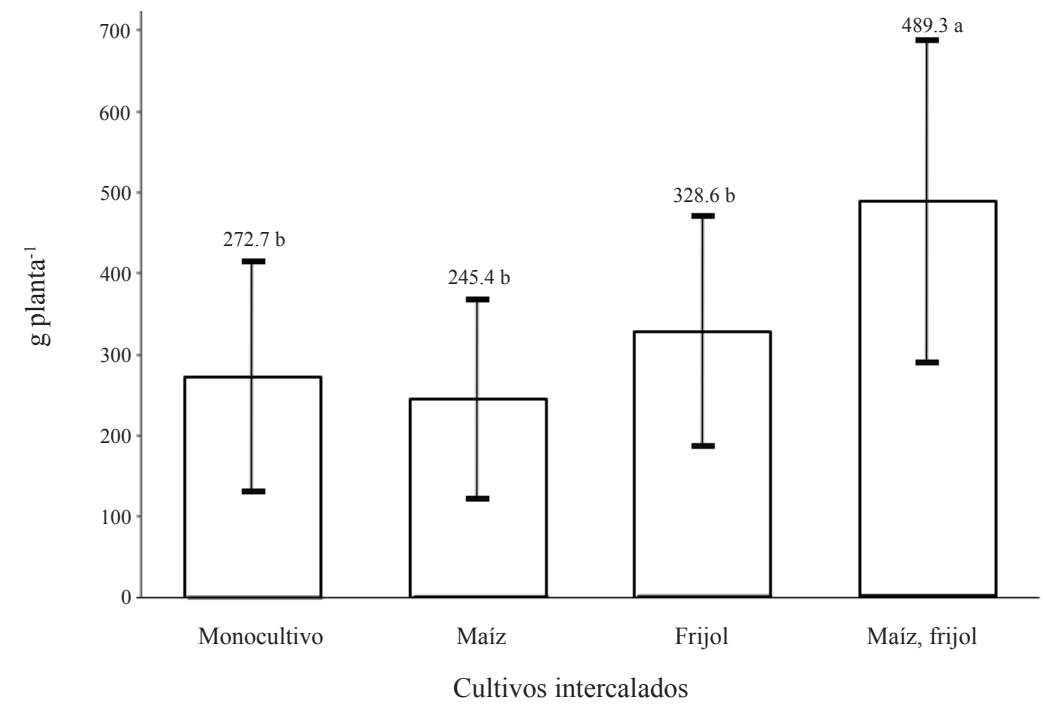

Figura 3. Rendimiento por planta de calabaza en monocultivo, asociado con maíz, con frijol y con ambos cultivos. * Letras distintas indican diferencias significativas, según la prueba de Tukey $(P \leq 0.05)$.

(Wang et al., 2010) ya que las tres plantas fueron domesticadas como policultivos (Zhang et al., 2014). Es decir, desde un inicio de su cultivación, las tres hermanas fueron adaptadas a la "convivencia".

La RET de 1.6 obtenida en el policultivo de las tres hermanas se debe a la sinergia en el desarrollo radicular de los tres cultivos (Zhang et al., 2014). Precisamente, maíz, frijol y calabaza se caracterizan por una diferente arquitectura radicular donde la mayor heterogeneidad se detecta en cuanto al crecimiento vertical (Postma y Lynch, 2012): las raíces del frijol son las menos y las raíces de la calabaza las más profundas; por ende, las raíces de maíz están en el intermedio de los anteriores en términos de profundidad, pero demuestran el mayor crecimiento horizontal (Zhang et al., 2014). Naturalmente, la separación de las áreas radiculares no es absoluta sino que puede haber intersecciones (Postma y Lynch, 2012). También cambian durante un ciclo de producción ya que calabaza y frijol tienen ciclos productivos más cortos que el maíz, el que tiende a colonizar las áreas que antes había ocupado el frijol (Albino et al., 2015). Como consecuencia de su distinta arquitectura radicular, los tres cultivos también tienen diferentes estrategias de absorción de nutrientes del suelo. Estas diferencias, resultado de la domesticación en conjunto, resultan en poca competencia y facilitan

Cuadro 5. Rendimiento referenciado a niveles de producción comunes en la región Atlacomulco, Estado de México, de maíz, frijol y calabaza (frutos) en las siete combinaciones de policultivos experimentadas y suma de los rendimientos referenciados (total).

\begin{tabular}{|c|c|c|c|c|c|}
\hline & \multicolumn{3}{|c|}{ Rendimiento referenciado $^{\dagger}$} & \multirow{2}{*}{ Total } & \multirow{2}{*}{ RET $^{*}$} \\
\hline & Maíz & Frijol & Calabaza & & \\
\hline & 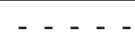 & $-\%-$ & $-\cdots$ & Puntos $\%$ & \\
\hline Maíz & 133.3 & & & 133.3 & 1.0 \\
\hline Maíz-Calabaza & 112.9 & & 60.8 & 173.7 & 1.6 \\
\hline Maíz-Frijol & 112.8 & 127.6 & & 240.4 & 1.9 \\
\hline Calabaza & & & 86.1 & 86.1 & 1.0 \\
\hline Frijol-Calabaza & & 39.5 & 116.5 & 156.0 & 1.7 \\
\hline Frijol & & 124.4 & & 124.4 & 1.0 \\
\hline Maíz-Frijol-Calabaza & 64.8 & 51.6 & 62.3 & 178.7 & 1.6 \\
\hline
\end{tabular}

${ }^{7} 7 \mathrm{Mg} \mathrm{ha}^{-1}=100 \%{ }^{\ddagger} 1.5 \mathrm{Mg} \mathrm{ha}^{-1}=100 \%{ }^{\S} 12 \mathrm{Mg} \mathrm{ha}^{-1}$ (frutos) $=100 \% .{ }^{\circledR}$ Razón equivalente de la tierra. 
la sobrecosecha en la milpa. El uso eficiente de los nutrientes disponibles favorece particularmente un rendimiento satisfactorio en suelos de baja fertilidad. Sin embargo, la sobrecosecha de maíz existe también en suelos bien nutridos (Zhang et al., 2014).

Aunque la competencia subterránea es mayor a la competencia superficial en los policultivos (Zhang et al., 2014), en la milpa existen también sinergias en el crecimiento superficial. Esta aplicó particularmente para maíz y frijol cuyo intercalado estimula la altura y producción de biomasa foliar de ambos (Bavec et al., 2005; Tembakazi y Lucas, 2002). Es la consecuencia de que cualquier asociación de maíz con una leguminosa captura más luz que los respectivos monocultivos (Albino et al., 2015; Francis y Decoteau, 1993) siempre que ambos cultivos no entren en competencia (García, 2002). Efectivamente, maíz y frijol tuvieron su mayor producción por planta asociados el uno con el otro (Figuras 1, 2). La RET de 1.9 en el policultivo de maíz y frijol es el resultado de una actividad fotosintética elevada en esta asociación que se refleja en la cosecha (Sullivan, 1998).

Otro factor que favorece la interacción entre maíz y frijol es la captura de nitrógeno atmosférico, ya que el frijol nodula más en asociaciones con el maíz, generando una ganancia neta de nitrógeno en asociaciones de maíz y una leguminosa (Gliessman, 1990). Por lo tanto, muchos productores del centro de México disminuyen sus inversiones en fertilizantes o abonos sembrando asociaciones de maíz y frijol o de maíz y haba (Vicia faba L.). En cambio, el policultivo maíz-frijol-calabaza se limita a regiones de alta población indígena (Altieri y Trujillo, 1987). Ya que la asociación de maíz y frijol generó mayor RET que la variante de las tres hermanas, para el Norte del Estado de México es recomendable seguir este patrón; es decir que se puede omitir la calabaza.

Sin embargo, asociando la calabaza con maíz (RET de 1.6), frijol (1.7) o ambos (1.6) para diversificar la producción sigue siendo una opción que también genera sobrecosecha. Como los rendimientos de todos los policultivos superaron los respectivos monocultivos, la selección del policultivo adecuado por el productor dependerá más bien de factores como características del área productiva, manejo preferido, costumbres, condiciones económicas, y disponibilidad de mano de obra (Altieri y Trujillo, 1987). En cuanto a la fertilidad del área de producción conviene considerar que la calabaza es el cultivo de las tres hermanas que es más sensible a escasa fertilidad, mientras que el frijol es el menos afectado por baja fertilidad (Itulya, 1980; Postma y Lynch, 2012).

Aparte de las ventajas productivas destaca también la alta resistencia de la milpa a perturbaciones externas, un hecho muy conveniente para enfrentar el cambio climático. Adicionalmente, la captación de $\mathrm{CO}_{2}$ atmosférico por plantas y su consecuentemente integración a la materia orgánica del suelo es una estrategia promisoria para atenuar el cambio climático. Los policultivos no solo superan monocultivos en términos productivos, sino también en su potencial de captar $\mathrm{CO}_{2}$. Particularmente la calabaza con su alta producción de biomasa superficial y subterránea tiene gran potencial en este contexto (Wang et al., 2010).

\section{CONCLUSIONES}

- La alta resiliencia de policultivos enfrentando perturbaciones externas es un amplio consenso en la comunidad científica y reconocida por muchos productores. Los policultivos tradicionales como la milpa son apreciados por su alta agrobiodiversidad que puede ser una herramienta esencial en la adaptación de los campos productivos al cambio climático. Pese a ello, persiste la duda por si la milpa todavía puede competir con los monocultivos modernos en términos productivos.

- En la presente investigación se compararon todas las posibles combinaciones de policultivos de maíz, frijol y calabaza (incluyendo los monocultivos de cada cultivo). De estos tratamientos, la combinación de maíz y frijol generó la mayor razón equivalente de la tierra (RET). Precisamente, resultó en una RET de 1.9. En otras palabras, maíz y frijol asociados produjeron $90 \%$ más cosecha por área que en sus respectivos monocultivos puesto que ambos cultivos asociados mostraron mayor cosecha por planta que en sus monocultivos. Este resultado encaja con el concepto de la sobrecosecha en asociaciones de maíz y leguminosas. Se demostró también que la inclusión de la calabaza a este policultivo (como es el caso en la clásica milpa), disminuye el rendimiento por planta de maíz y frijol, pero todavía genera una RET de 1.6. Consecuentemente, la asociación de maíz y frijol resultó ser el policultivo más productivo. Sin embargo, incluir la calabaza por motivos de diversificación no se considera una estrategia despreciable. También la cosecha de los policultivos de maíz y calabaza y de 
frijol y calabaza superó los respectivos monocultivos. - Se concluye que, al contrario de la tendencia contemporánea de reducir la agrobiodiversidad en los sistemas de producción, la diversificación es una estrategia funcional para incrementar la producción de los campos de maíz. Sí existe la necesidad de adaptar la milpa, un sistema de producción tradicional altamente diverso, a los retos del presente. Sin embargo, esta adaptación debería enfocarse más en cuestiones como la facilitación de su manejo; en términos productivos, la milpa supera los monocultivos de maíz.

\section{LITERATURA CITADA}

Agboola, A. A. and A. A. Fayemi. 1971. Preliminary trials on the intercropping of maize with different tropical legumes in Western Nigeria. J. Agric. Sci. 77: 219-225. doi: 10.1017/ S0021859600024345.

Aguilar, J., C. Illsley y C. Marielle. 2003. Los sistemas agrícolas de maíz y sus procesos técnicos. pp. 83-122. In: G. Esteva y C. Marielle (eds.). Sin maíz no hay país. CONACULTA. Ciudad de México.

Albino G., R., A. Turrent F., J. I. Cortés F., M. Livera M. y M. C. Mendoza C. 2015. Distribución de raíces y de radiación solar en el dosel de maíz y frijol intercalados. Agrociencia 49: 513-531.

Ali, Q. and M. Ashraf. 2011. Induction of drought tolerance in maize (Zea mays L.) due to exogenous application of trehalose: Growth, photosynthesis, water relations and oxidative defense mechanism. J. Agron. Crop Sci. 19: 258-271. doi: 10.1111/j.1439-037X.2010.00463.x

Altieri S., M. A. 1994. Bases agroecológicas para una producción agrícola sustentable. Agric. Tec. 54: 371-386.

Altieri, M. A. 2004. Linking ecologists and traditional farmers in the search for sustainable agriculture. Front. Ecol. Environ. 2: $35-42$.

Altieri, M. A. 2009a. Agroecology, small farms and food sovereignty. Mon. Rev. 61: 102-112. doi: 10.14452/MR-06103-2009-07_8.

Altieri, M. A. 2009b. La agricultura moderna: Impactos ecológicos y la posibilidad de una verdadera agricultura sustentable. University of California, Berkeley, Department of Environmental Science, Policy and Management. Berkeley, CA, USA.

Altieri, M. A. and J. Trujillo. 1987. The agroecology of corn production in Tlaxcala, Mexico. Hum. Ecol. 15: 189-220. doi: 10.1007/BF00888380.

Altieri, M. A. and P. Koohafkan. 2008. Enduring farms: Climate change, smallholders and traditional farming communities. Third world network. Penang, Malasia.

Altieri, M. A., D. K. Letourneau, and J. R. Davis. 1983. Developing sustainable agroecosystems. BioScience 33: 45-39. doi: $10.2307 / 1309244$

Altieri, M. A., F. R. Funes-Monzote, and P. Petersen. 2011. Agroecologically efficient agricultural systems for smallholder farmers: Contributions to food sovereignty. Agron. Sustain. Dev. 32: 3-15. doi: 10.1007/s13593-011-0065-6.
Augé, R. 2001. Water relations, drought and vesicular-arbuscular mycorrhizal symbiosis. Mycorrhiza 11: 3-42.

Bavec, F., U. Živec, S. Grobelnik M., M. Bavec, and L. Radics. 2005. Competitive ability of maize in mixture with climbing bean in organic farming. http://orgprints.org/4214 (Consulta: marzo 2, 2017).

Biggs, R. and P. Webb. 1986. Effects of enhanced UV-B radiation on yield, and disease incidence and severity for wheat under field conditions. pp. 303-311. In: R. C. Worrest and M. M. Caldwell (eds.). Stratospheric ozone reduction, solar ultraviolet radiation and plant life. Springer. Berlin and Heidelberg, Alemania.

CONAGUA (Comisión Nacional del Agua). 2016. Datos históricos de la estación meteorológica 15003, Almoloya de Juárez. http://smn1.conagua.gob.mx/index.php?option=com content\&view $=$ article $\&$ id $=189 \& \mathrm{tmpl}=$ component $\quad$ (Consulta: julio 6, 2016).

Dempewolf, H., R. Eastwood, L. Guarino, C. Khoury, J. Müller, and J. Toll. 2014. Adapting agriculture to climate change: A global initiative to collect, conserve, and use crop wild relatives. Agroecol. Sust. Food Syst. 38: 369-377. doi: 10.1080/21683565.2013.870629.

Dwivedi, S. L., K. L. Sahrawat, H. D. Upadhyaya, and R. Ortiz 2013. Food, nutrition and agrobiodiversity under global climate change. pp. 1-128. In: D. L. Sparks (ed.). Advances in Agronomy. Academic Press. Waltham, MA, USA. doi: 10.1016/B978-0-12-407686-0.00001-4.

Ebel, R. and J. A. Castillo-Cocom. 2012. X-Pichil: From traditional to "modern" farming in a Maya community. Memories of the VIII International Conference on Sustainable Agriculture, Environment and Forestry. Roma, Italia.

Ebel, R., M. J. Méndez A., E. E. Brito E. y H. Calix D. 2013. Producción agroecológica de chile habanero en su asociación con la pitahaya. pp. 35-58. In: R. Ebel (ed.). Producción extensiva de chile habanero. Académica Española. Saarbrücken, Alemania.

Francis, R. and D. R. Decoteau. 1993. Developing an effective southernpea and sweet corn intercrop system. HortTechnology 3: $178-184$.

Frison, E. A., J. Cherfas, and T. Hodgkin. 2011. Agricultural biodiversity is essential for a sustainable improvement in food and nutrition security. Sustainability 3: 238-253. doi: 10.3390/ su3010238.

Führer, J. 2003. Agroecosystem responses to combinations of elevated $\mathrm{CO}_{2}$, ozone, and global climate change. Agric. Ecosyst. Environ. 97: 1-20. doi: 10.1016/S0167-8809(03)00125-7.

García B., L. 2002. Plant-plant interactions in tropical agriculture. pp. 12-58. In: J. H. Vandermeer (ed.). Tropical agroecosystems. CRC Press. Boca Ratón, FL, USA. doi: 10.1201/9781420039887.

García M., E. y Z. Falcón G. 1980. Nuevo atlas porrúa de la República Mexicana. Edición Porrúa. Ciudad de México.

Gliessman, S. R. 1977. A biotechnological module for sustained yield agriculture in the humid lowland tropics. Intecol Newsl. 7: 173-185.

Gliessman, S. R. 1985. Multiple cropping systems: A basis for developing an alternative agriculture. pp. 67-83. In: US Congress Office of Technology Assessment. Innovative biological technologies for lesser developed countries: workshop proceedings. Congress of the USA. Washington, DC, USA. 
Gliessman, S. R. 1990. Applied ecology and agroecology: Their role in the design of agricultural projects for the humid Tropics. pp. 33-47. In: F. Montagnini and R. Goodland (eds.). Race to save the tropics: Ecology and economics for a sustainable future. Island Press. Washington, DC, USA.

Guzman, G. y A. Alonso. 2000. Las rotaciones y las asociaciones en el manejo de control de plagas y enfermedades. Hoja divulgativa 4.2/100. Comité Andaluz de Agricultura Ecológica. Sevilla, España.

Hernández X., E. 1995. La milpa en Yucatán: Un sistema de producción agrícola tradicional. Colegio de Postgraduados. Montecillo, México.

INIFAP (Instituto Nacional de Investigaciones Forestales, Agrícolas y Pecuarias). 2016. Datos meteorológicos del año 2015 de la estación Arroyo, Almoloya de Juárez. dehttp://clima.inifap.gob.mx/lnmysr/Estaciones/ ConsultaDiarios $15 \mathrm{Min}$ ? Estado $=10 \&$ Estacion $=48020$ (Consulta: julio 12, 2016).

Itulya, F. M. 1980. The influence of intercropping on growth and yield of summer squash (Cucurbita pepo L.), Mung bean (Phaseolus aureus Roxb.), and Pinto bean (Phaseolus vulgaris L.). University of Arizona. Tucson, AZ, USA.

Iverson, A. L., L. E Marín, K. K. Ennis, D. J. Gonthier, B. T. Connor, J. L. Remfert, J. Cardinale, and I. Perfecto. 2014. Do polycultures promote win-wins or trade-offs in agricultural ecosystem services? A meta-analysis. J. Appl. Ecol. 51: 1593-1602. doi: 10.1111/1365-2664.12334.

Jones, P. and P. K. Thornton, 2003. The potential impacts of climate change on maize production in Africa and Latin America in 2055. Glob. Environ. Change 13: 51-59. doi: 10.1016/S09593780(02)00090-0.

Khan, Z. R., C. A. O. Midega, D. M. Amudavi, A. Hassanali, and J. A. Pickett. 2008. On-farm evaluation of the 'push-pull' technology for the control of stemborers and striga weed on maize in western Kenya. Field Crops Res. 106: 224-233.

Lewandowski, S. 1987. Diohe'ko, the three sisters in seneca life: Implications for a native agriculture in the finger lakes region of New York State. Agricul. Hum. Values 4: 76-93. doi: 10.1007/BF01530644.

Liebman, M. 1997. Sistemas de policultivos. pp. 191-202. In: M. Altieri. Agroecología: Bases científicas para una agricultura sustentable. CIED. Lima, Perú.

Mariaca M., R. 2011. La milpa en el sur de México. Ecofronteras 42: $22-26$.

Mera O., L. M., R. A. Bye B., C. Villanueva V. y A. Luna M. 2011. Documento diagnóstico de especies cultivadas de Cucurbita en México. SAGARPA, SINAREFI, SNICS. México, D. F.
Molina A., M. F., J. L. Chávez S., A. Gil M., P. A. López, E. Hernández R. y E. Ortiz T. 2016. Eficiencias productivas de asociaciones de maíz, frijol y calabaza (Curcurbita pepo L.) intercaladas con árboles frutales. Phyton 85: 36-50.

Morton, J. 2007. The impact of climate change on smallholder and subsistence agriculture. Proc. Natl. Acad. Sci. USA104: 19680-19685. doi:10.1073pnas.0701855104.

Patterson, D. T. 1995. Weeds in a changing climate. Weed Sci. 43: 685-701.

Pimentel, D. 1993. Climate changes and food supply. Forum Appl. Res. Public Policy 8: 54-60.

Postma, J. A. and J. P. Lynch. 2012. Complementarity in root architecture for nutrient uptake in ancient maize/bean and maize/bean/squash polycultures. Ann. Bot. 110: 521-534. doi: 10.1093/aob/mcs082.

Real Academia Española. 2014. Milpa. In: Diccionario de la lengua española, edición 2014. http://dle.rae.es/?id=PGZxeer (Consulta: marzo 7, 2017).

Risch, S. J. and M. K. Hansen. 1982. Plant growth, flowering phenologies, and yields of corn, beans and squash grown in pure stands and mixtures in Costa Rica. J. Appl. Ecol. 19: 901-916. doi: 10.2307/2403292.

Root, R. 1973. Organization of a plant-arthropod association in simple and diverse habitats: The fauna of collards (Brassica oleracea). Ecol. Monogr. 43: 95-124.

Santalla, M., A. P. Rodino, P. A. Casquero, and A. M. de Ron. 2001. Interactions of bush bean intercropped with field and sweet maize. Eur. J. Agron. 15: 185-196. doi: 10.1016/S11610301(01)00104-6.

Swaminathan, M. and P. C. Kesavan. 2012. Agricultural research in an era of climate change. J. Agric. Res. 1: 3-11. doi:10.1007/ s40003-011-0009-z.

Sullivan, P. 1998. Intercropping principles and production Practices. http://www.iatp.org/files/Intercropping_Principles_and_ Production_Practi.htm (Consulta: marzo 5, 2017).

Tembakazi S., T. and E. O. Lucas. 2002. The effect of planting combinations and weeding on the growth and yield of component crops of maize/bean and maize/pumpkin intercrops. J. Agric. Sci. 138: 193-200. doi: 10.1017/S0021859601001861

Wang, Q., Y. Li, and A. Alva. 2010. Cropping systems to improve carbon sequestration for mitigation of climate change. J. Environ. Prot. 3: 207-215. doi: 10.4236/jep.2010.13025

Zhang, C., J. A. Postma, L. M. York, and J. P. Lynch. 2014. Root foraging elicits niche complementarity-dependent yield advantage in the ancient 'three sisters'(maize/bean/squash) polyculture. Ann. Bot. 191: 1719-1733. doi: 10.1093/aob/ mcu191. 